\title{
The certitude of a global sea level acceleration during the satellite altimeter era
}

DOI: https://doi.org/10.1515/jogs-2020-0101

Received December 15, 2019; accepted January 29, 2020

\begin{abstract}
Recent studies reported a uniform global sea level acceleration during the satellite altimetry era (19932017) by analyzing globally averaged satellite altimetry measurements. Here, we discuss potential omission errors that were not thoroughly addressed in detecting and estimating the reported global sea level acceleration in these studies. Our analyses results demonstrate that the declared acceleration in recent studies can also be explained equally well by alternative kinematic models based on previously well-established multi-decadal global mean sea level variations of various origins, which suggests prudence before declaring the presence of an accelerating global mean sea level with confidence during the satellite altimetry era.
\end{abstract}

Keywords: Satellite altimetry; Global sea level acceleration; Tide gauge; Omission error; Global sea level budget; Climate change.

"Nothing is always absolutely so." Sturgeon, The Claustrophile, 1956.

\section{Introduction}

Evidence for the global mean sea level, GMSL, rising faster during the 20th and $21^{\text {st }}$ centuries with global warming is an important indicator in assessing anthropogenic contributions to the climate change mechanisms.We examined various components of the global sea level variability, whose presence makes it difficult to detect the global sea level acceleration that may be due to natural internal climate variability or anthropogenic causes or both. We analyzed monthly and globally averaged satellite altimetry, SA, global sea level time series to demonstrate that one of

\footnotetext{
*Corresponding Author: H. Bâki İz: Division of Geodetic Science, School of Earth Sciences, The Ohio State University, Columbus, Ohio, USA

C.K. Shum: Division of Geodetic Science, School of Earth Sciences, The Ohio State University, Columbus, Ohio, USA
}

the most important approaches to earliest possible detection of a significant GMSL acceleration lies in recognizing and separating the effect of natural internal forcing of the oceans compounded with the variability of astronomical origin that are not limited to the SA era. We also quantified unusually highly autocorrelated noise in the SA time series, which were not rigorously addressed in recent studies.

In the past, several studies investigated the presence of a global acceleration during the $20^{\text {th }}$ century in sea-level rise with mixed results (see İz, 2017 for references). More recently, İz (2017) verified that there was no statistically significant ${ }^{1}$ observational evidence for a GMSL acceleration during the $20^{\text {th }}$ century by analyzing monthly mean sea level data at globally distributed tide gauge, TG, stations with long records. However, the absence of GMSL acceleration during the $20^{\text {th }}$ century cannot be cast into the future with confidence given the recent observational evidence for an acceleration at globally distributed TG stations determined using a broken trend model by İz et al. (2018) from TG measurements from 1993 to present.

Along these lines, Yi, et al. (2015) also reported an increase in GMSL over $1.4 \mathrm{~mm} / \mathrm{yr}$. since 2010 with respect to background sea level trend of $3 \mathrm{~mm} / \mathrm{yr}$. by analyzing $\mathrm{SA}$ data. This rate of change in sea level rise is equivalent to an average acceleration of $0.28 \mathrm{~mm} / \mathrm{yr}^{2}$, which is extremely fast for such a brief time span. Davis and Vinogradova (2017) reported an acceleration along the east coast of North America up to $0.3 \mathrm{~mm} / \mathrm{yr}^{2}$ through the analysis of SA time series. Dieng et al. (2017) determined an increase of about $0.8 \mathrm{~mm} / \mathrm{yr}$. in the GMSL velocity since 2004, which is equivalent to an average acceleration of $0.062 \mathrm{~mm} / \mathrm{yr}^{2}$. However, these studies did not report uncertainties about the estimated velocities. Moreover, there is no evidence that these studies accounted for the effect of highly autocorrelated noise of the SA measurements in their analyses, which would have caused Type I error in testing the statistical significance of their findings had they carried out.

\footnotetext{
1 We will be referring to a $p$-value less than or equal to $0.05 \alpha$ level for statistical significance throughout this study.
} 
Most recently Nerem, et al. (2018) reported $0.084 \pm 0.025 \mathrm{~mm} / \mathrm{yr}^{2}$ GMSL acceleration since 1993 inferred from the globally averaged SA time series. This study recognized and reported correcting the effect of autocorrelation but without any detail and conducted a rudimentary prediction ${ }^{2}$ for the future global sea level rise. Soon after, Ablain et al. (2019) reported another estimate of GMSL acceleration, $0.12 \pm 0.07 \mathrm{~mm} / \mathrm{yr}^{2}(90 \%$ confidence level), with a full variance covariance matrix, V/C, encapsulating various SA instrumental errors, commission errors, neither corrected nor modeled in their estimation but to recalibrate the standard errors of the GMSL acceleration estimated using a non-optimal ordinary least squares, OLS, solution, for their omission ${ }^{3}$.

In contrast to SA, TG measurements sample only local and regional sea level variations. Their limited global distributions are prohibitive in making a conclusive inference about the GMSL trend and acceleration. However, tide gauges can serve effectively as a consilience tool to verify SA findings about the GMSL rise. In a recent study, İz et, al. (2019) analyzed sea level trends at globally distributed 27 TG stations using a broken trend model that accommodates changes both at sea level velocities and recurring interannual, decadal, and interdecadal sea level variations before and during the SA era. Inferred sea level accelerations in this study are less prone to a potential omission errors of multidecadal sea level variations at these stations because of the century long time span compared to accelerations estimated from shorter SA time series. The reported estimates for accelerations at globally distributed TG stations with century long records turned out to be preponderantly smaller than the accelerations reported using satellite altimetry data (ibid.). In this study, we model such low frequency sea level variations inferred from globally distributed TG measurements but not included in recent studies in analyzing SA measurements in detecting and estimating the global sea level acceleration.

In the following sections, we first give a brief description of the global SA monthly time series to be used in this study. A baseline kinematic model is then introduced to estimate global velocity and acceleration using OLS from the globally and monthly averaged SA time series. The ap-

2 Nerem, et al. (2018) uses the term projection in their publications. Kinematic models used in the context of future sea level rise predict as opposed to project. See the commentary by Bray and Storch (2009) for the importance of the difference between prediction and projection. 3 It is statistically inappropriate to ignore a properly formed V/C matrix in estimating GMSL acceleration using GLS procedure, but instead use an OLS solution then correct for the effect of omission without any justification. proach mirrors the quadratic models deployed by Nerem et al., (2018) and Ablain et al., (2019). The residual analysis of the OLS led to the necessity of modelling/eliminating for the effect of the autocorrelations. We then introduced a new model based on differencing subsequent SA measurements to eliminate first order autoregressive residuals. We also established another statistical model to be used by a generalized least squares, GLS, procedure as an alternative approach, which is also suitable for rigorous predictions, for cross checking the results of the differenced model and rigorously estimated velocity and acceleration parameters and their statistics without eliminating but discounting them. Finally, we built and assessed alternative kinematics models with a jerk, and with low frequency decadal and multidecadal sea level variations in explaining GMSL anomalies during the SA era.

\section{Global Mean Sea Level Data}

This study utilizes monthly averaged GMSL data, shown in Fig. $(1)^{4}$, which were generated using the Integrated Multi-Mission Ocean Altimeter Data for Climate Research (GSFC, 2017). It combines Sea Surface Heights from TOPEX/Poseidon, Jason-1 and OSTM/Jason-2 with all systematic errors corrected and placed onto a georeferenced orbit (Beckley, et al., 2016).

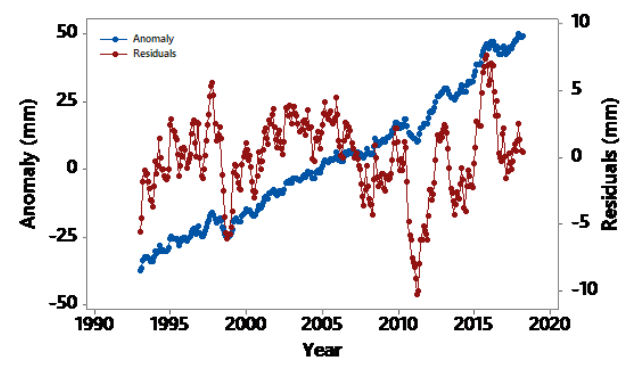

Fig. 1. Globally and monthly averaged sea level anomalies as observed by Satellite Altimetry (Blue), superimposed with the residuals of the OLS.

4 A detailed plot indicating mission periods is available at NASA's PODAAC website https://podaac.jpl.nasa.gov/ 


\subsection{Estimating GMSL trend and acceleration using OLS from globally averaged SA time series and residual analysis}

We will use the following kinematic and statistical model as a strip down start-up model for estimating the global sea level velocity and acceleration. It is the kinematic version of the quadratic fits used by Nerem et al., (2018) and Ablain et al., (2019), with the only difference in assuming the disturbances being white, as opposed to being first order autoregressive, i.e.,

$$
h_{t}=h_{t_{0}}+v\left(t-t_{0}\right)+\frac{1}{2} a\left(t-t_{0}\right)^{2}+u_{t} \quad u_{t} \sim \operatorname{iid}\left(0, \sigma_{u}^{2}\right)
$$

In this representation, $h_{t}$ denotes a monthly globally averaged SA observations at epochs $t=1 \ldots n$, where $n$ is the number of months. The intercept $h_{t_{0}}$ is the reference height of the globally and monthly averaged sea level defined at the reference epoch $t_{0}$ chosen to be in the middle of the time series. The intercept $h_{t_{0}}$ is estimated together with the global uniform velocity and uniform acceleration parameters, $v, a$, respectively. In eqn. (1), it is assumed that the errors of monthly averaged SA measurements at epochs $t$, denoted by $u_{t}$ are identically and independently distributed, iid, with an expected value of zero, i.e. random noise.

The solution of this model using OLS explains $98 \%$ of the variation $\left(\operatorname{Adj} R^{2}=98.4\right)$ in the global SA monthly time series, which is an impressive fit for such a simple model. The estimated velocity and acceleration parameters are listed in Table 1 under Solution I. Model statistics are equally impressive and statistically significant. Yet, the residuals displayed on Figure 1 exhibit non-random excursions that are not explained by the model, which are in contradiction with the extremely precise standard errors of the estimated parameters and white noise assumption.

Figure 2, the correlogram of the residuals explains the underlying cause of underestimating the standard errors of the estimates and nonrandom excursions in residuals (Neter et al, 1996). It is also a testament of positive autocorrelated noise. The autocorrelations of the residuals are statistically significant down to 8 monthly lags. Although positive autocorrelations are recognized in $\mathrm{TG}$ time series occasionally in sea level studies (İz et al., 2012), what is striking in this case, is their magnitudes in the residuals of the global SA time series. In particular, the first order autocorrelation AR(1), with its estimated correlation coefficient $\hat{\rho}_{1}=0.93$ is extremely large approaching to a unit root process (random walk) as compared to the first order autocorrelations observed of the TG time series within the range $0.2-0.4$, (İz, 2014). It is also surprising that the autocorrelation was recognized by Nerem et al. (2018) but its magnitude was not reported. Similarly, Ablain et al., (2019) discussed measurement errors with "time correlation" in lengthy detail but provided no information for unmodelled autocorrelations in their OLS residuals. A near unit root process in sea level variations absolves the GMSL trend and a potential acceleration from being deterministic, hence makes GSML variations difficult to predict.

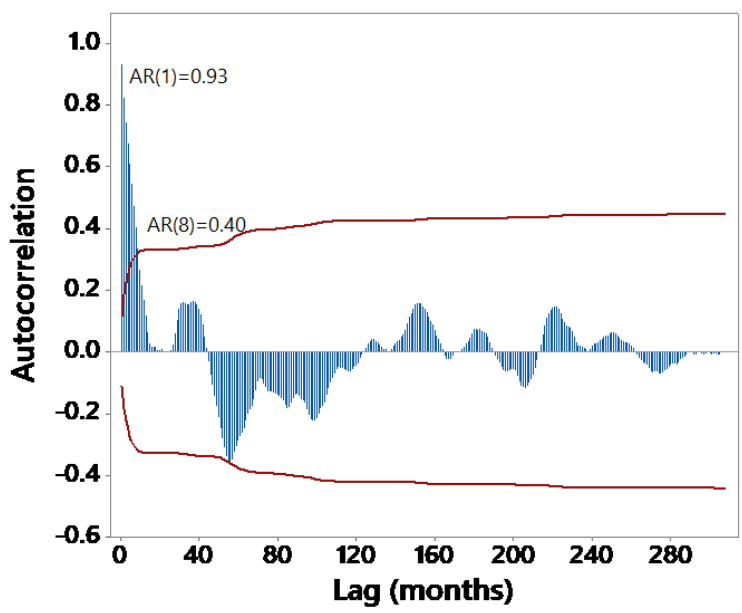

Fig. 2. Correlogram of the residuals. Confidence interval is $95 \%$.

In addition to measurement errors, progression of random errors can also create autoregressive disturbances as we will demonstrate in the following section. Excessive smoothing of the records to remove high frequency variations also induces autocorrelations. In any case, the effect of the high autocorrelation must be accounted for not only in estimating velocity and acceleration of the GMSL rise and their statistical significance, but also in sea level predictions (İz, 2019). In GMSL time series, it is also important to identify if the autocorrelations are the intrinsic property of the series as part of a physical system. An intrinsic high autocorrelation in the series can generate long term persistence, and vulnerability of the GMSL to the random innovations/shocks of natural origin, which may create unpredictable changes as in the progression of the GMSL rise. Fortunately, the correlogram shown in Figure 2 does not reveal any statistically significant long-term persistence at $95 \%$ confidence level despite the large lag 1 autocorrelation.

Residuals in Figure 1 exhibit statistically significant changes up to lag 8 months and their magnitudes are sizeable comparable to the monthly anomalies. What is 
Table 1. Model solutions. All uncertainties are 1 $\sigma$. N/A: Not Applicable. N/E: Not Estimable. H-L: Hildreth-Lu. SE: Standard Error, Amp.: Amplitude.. *Jerk.

\begin{tabular}{llllllll}
\hline Solution & Model & $\begin{array}{l}\text { Solution } \\
\text { Method }\end{array}$ & $\begin{array}{l}\text { Velocity } \\
(\mathrm{mm} / \mathrm{yr})\end{array}$ & $\begin{array}{l}\text { Acceleration } \\
\left(\mathrm{mm} / \mathrm{yr}^{2}\right)\end{array}$ & $\begin{array}{l}\text { Amp. } \\
(\mathrm{mm})\end{array}$ & $\begin{array}{l}\text { SE } \\
(\mathrm{mm})\end{array}$ & $\begin{array}{l}\text { AR(1) } \\
\rho\end{array}$ \\
\hline Nerem et al. 2018 & v, a & AR(1), How? & $2.90 \pm 0.40$ & $0.084 \pm 0.025$ & N/A & N/A & N/A \\
Ablain et al. 2019 & v, a & OLS, Error V/C & $3.35 \pm 0.24$ & $0.120 \pm 0.040$ & N/A & N/A & 0.00 \\
I (1993-2017) & v, a & OLS & $3.22 \pm 0.02$ & $0.098 \pm 0.007$ & N/A & 3.01 & 0.00 \\
II (1993-2017) & v, a & OLS (H-L) & $3.13 \pm 0.12$ & $0.124 \pm 0.037$ & N/A & 1.06 & 0.93 \\
III (1993-2017) & v, a & GLS & $3.27 \pm 0.10$ & $0.081 \pm 0.030$ & N/A & 2.89 & 0.93 \\
IV (1993-2017) & v, j & OLS (H-L) & $2.76 \pm 0.27$ & $0.0324 \pm 0.0154 *$ & N/A & 1.07 & 0.93 \\
V (1993-2017) & v, P=60yr & OLS (H-L) & $3.14 \pm 0.12$ & N/E & $12.7 \pm 3.8$ & 1.06 & 0.93 \\
VI (1993-2017) & v, P=74yr & OLS (H-L) & $3.14 \pm 0.12$ & N/E & $18.7 \pm 5.6$ & 1.06 & 0.93 \\
VII (1993-2017) & v, P=56yr & OLS (H-L) & $3.14 \pm 0.12$ & N/E & $11.2 \pm 3.3$ & 1.06 & 0.93 \\
VIII (1993-2017) & v, P=37yr & OLS (H-L) & $3.16 \pm 0.12$ & N/E & $5.8 \pm 1.8$ & 1.06 & 0.93 \\
\hline
\end{tabular}

equally important however is their origin and their magnitudes. This is the topic of the next section.

\section{The origin of the autoregressive disturbances}

Nerem et al (2018) study identifies El Niño-Southern Oscillation, ENSO, and Pacific Decadal Oscillation, PDO, as the origin of some of the unmodeled GMSL variation during the SA period and removes their effects by computing a correction via a joint cyclo-stationary empirical orthogonal function (CSEOF) analysis of altimeter GMSL, GRACE land water storage, and Argo-based thermosteric sea level from 2005 to present. Instead, we will use Multivariate ENSO Index, MEI, which combines both oceanic and atmospheric variables, and facilitates assessing the effect of ENSO in a single index. The index gives real-time indications of ENSO intensity through historical analysis of various data (NOAA, 2019).

OLS residuals and MEI time series are shown in Figure 3. Despite the visually close correlation between the two series, the maximum cross-correlation of MEI with OLS residuals is 0.52, which occurs with a time lag of 4 months (Figure 4). In other words, MEI is not the only unexplained variation in the globally averaged SA time series. In other words, the underlying cause of autoregressive disturbances includes both the ENSO effect at a global scale (Nerem et al., (2018) and time correlated instrumental error of Ablain et al (2019). Although the autocorrelation still dictates the need for the correcting the ENSO effect as it was carried out by Nerem et al., (2018), there is a simpler and more effective approach, which not only eliminates the impact of the ENSO but also reduces the effect of other random variations in the GMSL time series. This approach will also effectively eliminate the impact of the GMSL variations due to the AR(1) in estimating the GMSL trend and acceleration.

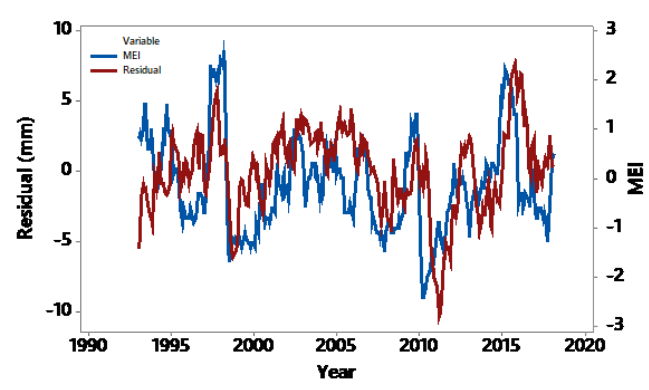

Fig. 3. MEI and Solution I monthly OLS residuals $(\mathrm{mm})$ during the SA period.

Figure 5 exhibits lag 1 differences of both series. From the figure it is evident that the first order differencing of monthly series is better than correcting the series for the signature of the ENSO and the instrumental errors altogether because it eliminates the random excursions induced by the $\mathrm{AR}(1)$ process effectively thereby, reducing the remaining variability in the series. Incorporating lag 1 differencing properly in modelling globally averaged SA measurements in estimating trend and acceleration in GMSL is the topic of the following section. 


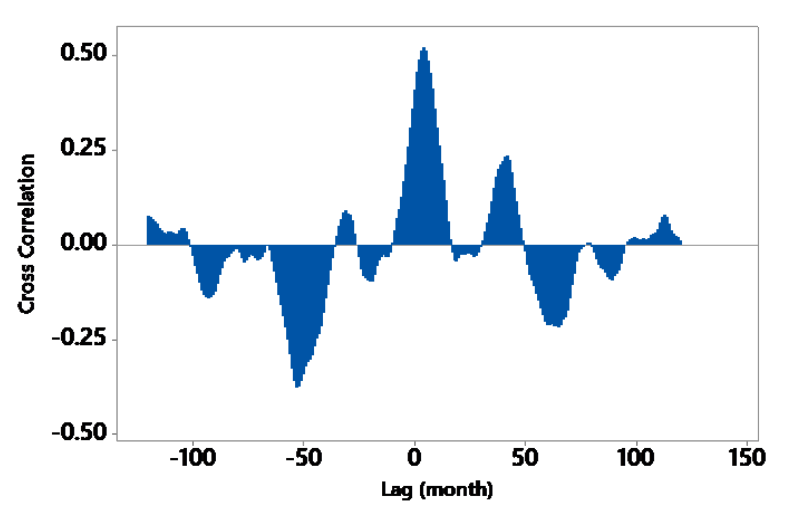

Fig. 4. Cross correlation of MEl and residuals. Maximum cross correlation 0.52 occurs at lag 4 .

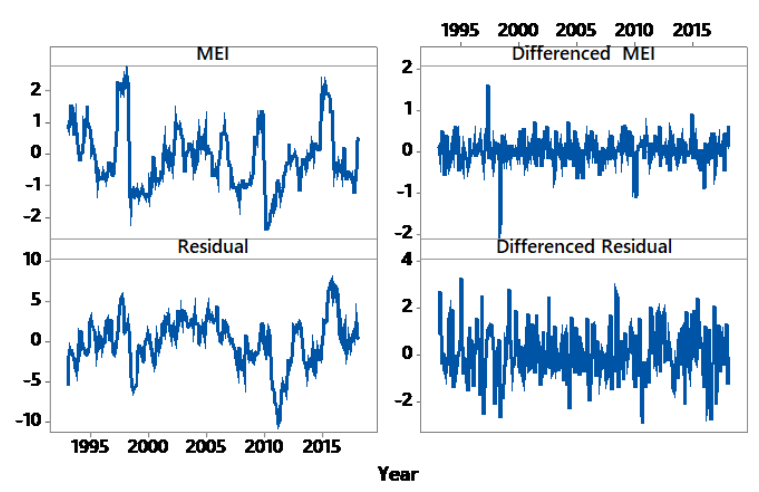

Fig. 5. Differenced (lag 1) MEI and Solution I monthly residuals (mm) listed in Table 1. Both series exhibit AR(1), 0.94 and 0.93 for the MEI and SA OLS residuals, respectively.

\section{Estimation of the GMSL trend and acceleration using differenced global SA time series}

Recognizing AR(1) properties of the disturbances discussed in the earlier section, eqn.(1) is recast as,

$$
h_{t}=h_{t_{0}}+v\left(t-t_{0}\right)+\frac{1}{2} a\left(t-t_{0}\right)^{2}+\varepsilon_{t}
$$

In comparison to eqn. (1), disturbances, $\varepsilon_{t}$, are now autocorrelated up to the first order, i.e.,

$$
\varepsilon_{t}=\rho \varepsilon_{t-1}+u_{t} \quad 0 \leq|\rho|<1
$$

where $\rho$ refers to the first order autocorrelation coefficient. The white noise is $u_{t} \sim \operatorname{iid}\left(0, \sigma_{u}^{2}\right)$, and $\sigma_{u}^{2}$ is the variance of the purely random variations (innovations). In this representation, as before, a monthly globally averaged SA observation at an epoch $t$ is denoted by $h_{t}$. The time index $t=1 \ldots n$, where $n$ is the number of months and includes all the available globally averaged SA data. The intercept $h_{t_{0}}$ is the height of the globally averaged sea level defined at the reference epoch $t_{0}$ chosen to be in the middle of the time series, which is to be estimated together with the global velocity and acceleration, $v, a$, parameters respectively.

Now, we will represent any two consecutive monthly SA measurements as,

$$
\begin{gathered}
h_{t-1}=h_{t_{0}}+v\left(t_{t-1}-t_{0}\right)+a \frac{1}{2}\left(t_{t-1}-t_{0}\right)^{2}+\varepsilon_{t-1} \\
h_{t}=h_{t_{0}}+v\left(t_{t}-t_{0}\right)+a \frac{1}{2}\left(t_{t}-t_{0}\right)^{2}+\varepsilon_{t}
\end{gathered}
$$

Multiplying eqn. (4) with $\rho$ and subtracting from eqn. (5) gives,

$$
\begin{aligned}
& h_{t}-\rho h_{t-1}=\left[h_{0}-\rho h_{0}\right]+v\left[\left(t_{t}-t_{0}\right)-\rho\left(t_{t-1}-t_{0}\right)\right] \\
& +\frac{1}{2} a\left[\left(t_{t}-t_{0}\right)^{2}-\rho\left(t_{t-1}-t_{0}\right)^{2}\right]+u_{t}
\end{aligned}
$$

The disturbances of this observation equation are now free from the AR(1) effect, and OLS can still be used to estimate model parameters iteratively using various autocorrelation coefficients within the interval $0 \leq|\rho|<1$ to determine the one that minimizes the a posteriori variance of the unit weight (the standard error of the solution). Durbin-Watson statistic can also be used as a criterion to determine the correlation coefficient that gives the minimum correlation between adjacent disturbance terms (Neter et al., 1996). The approach we just described that uses standard error of the solution is known as the Hildreth $\mathrm{Lu}$ procedure (Hildreth, and $\mathrm{Lu}, 1960$ ), which will be abbreviated as $\mathrm{H}-\mathrm{L}$.

The estimates for the velocity and acceleration parameters using H-L procedure are shown in Table 1, as Solution II. The magnitudes of the velocity and acceleration estimates did not change markedly compared to the regular OLS (Solution I) as expected. Nonetheless, the corresponding standard error of the estimates are larger, but they are realistic reflections of the uncertainties of the estimates. Solution II residuals are now free from AR(1) effect as shown in Figure 6. Note that the listed standard error (RMSE, a posteriori variance of the unit weight) is smaller for Solution II because it refers to the white noise $u_{t}$ only, not to the red noise $\varepsilon_{t}$ as in the case of Solution I.

It is important to note that Solution II replicated the estimate for the acceleration reported by Ablain et al (2019) without going through their adjustments and generating an elaborate V/C matrix for time correlated instrumental drifts, biases and noises implemented in their models. Solution II 
also produced an estimate for the GMSL acceleration similar to the Nerem et al. (2018) solution without applying any corrections for ENSO as well as the effect of the eruption of Mount Pinatubo in (1991) on global sea level.

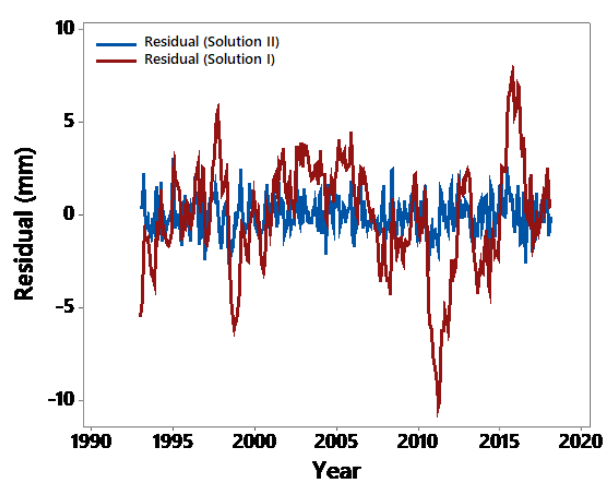

Fig. 6. Solution I and Solution II residuals. Note that Solution II residuals, in blue, are now free from the AR(1) process.

\section{Generalized least squares solution with an autoregressive $\mathrm{V} / \mathrm{C}$ matrix}

As an alternative to the statistical model and its solution using OLS with H-L procedure deployed in the previous section, autocorrelated SA measurements can also be rigorously modelled through a variance-covariance, $\mathrm{V} / \mathrm{C}$, matrix, which effectively represents the impact of the autocorrelations in estimating and predicting sea level variations and can be used in solving for the GMSL sea level velocity and acceleration parameters using generalized least squares, GLS. The formulation was used in the sea level studies (Iz, 2018) and serves as a cross check for the earlier model of Solution II, OLS with (H-L). It also offers advantages in GMSL predictions.

As before, we will consider the following kinematic model.

$$
h_{t}=h_{t_{0}}+v\left(t-t_{0}\right)+a \frac{1}{2}\left(t-t_{0}\right)^{2}+\varepsilon_{t}
$$

The disturbances $\varepsilon_{t}$, as before, are autocorrelated as described by eqn. (3),
In this formulation, we model the $n \times n \mathrm{~V} / \mathrm{C}$ matrix of the first order autocorrelated disturbances, $\Sigma$, as,

$$
\Sigma=\sigma^{2} \cdot\left[\begin{array}{ccccc}
1 & \rho & \rho^{2} & \cdots & \rho^{T-1} \\
\rho & 1 & \cdots & \cdots & \rho^{T-2} \\
\vdots & \vdots & \vdots & \ddots & \vdots \\
\rho^{T-1} & \rho^{T-2} & \rho^{T-3} & \cdots & 1
\end{array}\right]
$$

(Iz, 2018a). The autocorrelation decreases for increasing time lag because $|\rho|<1$. The correlation between two random variables $\varepsilon_{t}$ and $\varepsilon_{t-\tau}$ is $\sigma^{2} \rho^{\tau}$, where $\tau$ is the time lag. The above patterned $\mathrm{V} / \mathrm{C}$ matrix has an analytical inverse, and is given by,

$$
\begin{aligned}
\Sigma^{-1} & =\frac{\sigma^{-2}}{1-\rho^{2}} \mathbf{P} \\
& =\left[\begin{array}{cccccc}
1 & -\rho & 0 & \cdots & 0 & 0 \\
-\rho & 1+\rho^{2} & -\rho & \cdots & 0 & 0 \\
0 & -\rho & 1+\rho^{2} & \cdots & 0 & 0 \\
\vdots & \vdots & \vdots & \ddots & \vdots & \vdots \\
0 & 0 & 0 & \cdots & 1+\rho^{2} & -\rho \\
0 & 0 & 0 & \cdots & -\rho & 1
\end{array}\right]
\end{aligned}
$$

where $\mathbf{P}$ is the $n \times n$ weight matrix. In this model, the initial autocorrelation coefficient $\rho$ is estimated from the residuals of the ordinary least squares, OLS iteratively, and used in a generalized least squares, GLS, solution in forming the V/C matrix for the SA measurements. The residuals of the GLS solution are then used to calculate a new AR(1), which is then used in another GLS solution till there are no changes in the a posteriori variance of the unit weight (the standard error of the solution). Iterations result in an optimal AR(1) between adjacent disturbance terms to be used in the final GLS estimation and predictions.

We used this formulation to estimate velocity and acceleration parameters using GLS. Solution statistics are displayed in Table 1 as Solution III. Solution III produced comparable results to Solution II. It is important to note that we again replicated the same acceleration estimate reported this time by Nerem, et, al., (2018), without applying any corrections for ENSO, episodic variability driven by large volcanic enuptions, errors in the altimeter data, and potential drifts in the instruments over time.

So far, all our discussions can be lumped under the topic of quantifying the commission errors of various solutions. There may be other error sources, omission errors, which occur due to various contributors/confounders of physical origin on sea level variations that are ignored in the kinematic models in the previous sections, more importantly, in two studies recent studies by Nerem, et, al. (2019), and Ablain, et, al. (2018). This is the topic of the next section. 


\section{A recent jerk as opposed to a uniform acceleration in GMSL anomalies during the SA era}

Recall that the preponderant number of studies using TG measurement cited in the introduction section and recent literature did not find conclusively a global acceleration during the $20^{\text {th }}$ century. A global sea level acceleration was only reported recently by Nerem et al., (2018) and Ablain et al., (2019) using SA measurements during the SA era. The time lag for detecting a statistically significant acceleration during the last two decades can be attributed to the short time span of the SA time series or there may be other explanations. The acceleration may have started due to a recent innovation/shock experienced in sea level variations whose origin is yet to be investigated. Such a shock is akin to what we experience as a rapid change while driving a car or changing gears. We may experience a sudden movement if we push the gas pedal forcefully when we start the car. This rapid change in acceleration or velocity produces what is known as jerk. Kinematically, jerk is the second derivative of the velocity or the first derivative of acceleration with respect to time. İz et al. (2013) modeled, detected, and estimated such variable accelerations experienced at TG stations.

The following kinematic model includes, velocity, acceleration, and jerk parameters,

$$
h_{t}=h_{t_{0}}+v\left(t-t_{0}\right)+a \frac{1}{2}\left(t-t_{0}\right)^{2}+j \frac{1}{6}\left(t-t_{0}\right)^{3}+\varepsilon_{t}
$$

In this model, $j$ represents the jerk coefficient to be estimated. All the other model parameters were already defined by eqn. (7) and eqn. (3) together with their descriptions.

We estimated the unknown parameters in eqn. (10) using OLS with (H-L) procedure. However, the solution revealed that jerk and acceleration parameters cannot be estimated simultaneously because they are collinear ${ }^{5}$. But the jerk parameter can be estimated in the absence of the acceleration parameter. Under this scenario, jerk refers to a sudden change in a uniformly rising GMSL, the second derivative of the velocity, as opposed to a change in a uniformly accelerating GMSL. We then estimated only the jerk parameter, $\hat{j}$ using OLS with (H-L) procedure and determined it to be statistically significant (Table 1Solution IV). The results indicated that Solution IV is as good as Solution II, with an acceleration parameter only, i.e. they are

5 In the sense of a numerically strong linear relationship between the two parameters. interchangeable. While the estimated velocity with jerk is different than the estimated velocity with an acceleration, it is in exact agreement (within the round of errors) with the velocity estimate reported by IPCC, $2.8 \mathrm{~mm} / \mathrm{yr}$. with a confidence interval [2.1 to 3.5] during the 1993-2010 (Church et al., 2013). A jerk in global sea level can be a manifestation of a phenomenon, like the acceleration, whose origin is yet to be identified. Meanwhile, the equivalency between jerk and acceleration will inevitably have significant implications for global sea level predictions and of course in understanding global climate variability.

\section{A confounder: The 60-year period}

Sea level variations are multi-causal. Some of the effects are global isostatic adjustment, periodic changes in sea levels induced by wind, pressure, external forcing such as of lunar solar origin, and thermosteric effects of warming oceans, or they are eustatic in nature. The effects may be secular, episodic, transient, periodic at semi-annual, annual, interannual, decadal, or decadal and multidecadal time scales, all contributing to sea level anomalies. These effects are also local, regional, and could be global. Yet, the two recent studies that reported global sea level accelerations, by Nerem et al, (2018) and Ablain et al. (2019), ignored the contribution of these multidecadal effects in their analyses in detecting the GMSL acceleration. Moreover, Nerem et al. (2018) also claim that, we quote, While estimates of the impact of decadal variability on the 25-yr long time series are difficult to obtain, satellite altimetry is far less sensitive to decadal variability than tide-gauge measurements because of its superior global coverage.

Although this conjecture of decadal sea level variations being less sensitive at global scale is yet to be demonstrated, there are multitude of multidecadal effects whose presence were already established in TG series. For instance, the presence of a 60-year period in global sea level variation by a well-known study published in 2012, reported that:

...We find that there is a significant oscillation with a period around 60-years in the majority of the tide gauges examined during the 20th Century, and that it appears in every ocean basin. Averaging of tide gauges over regions shows that the phase and amplitude of the fluctuations are similar in the North Atlantic, western North Pacific, and Indian Oceans, while the signal is shifted by 10 years in the western South Pacific. The only sampled region with no apparent 60-year fluctuation is the Central/Eastern North Pacific. The phase of the 60-year oscillation found in the tide 
gauge records is such that sea level in the North Atlantic, western North Pacific, Indian Ocean, and western South Pacific has been increasing since 1985-1990. Although the tide gauge data are still too limited, both in time and space, to determine conclusively that there is a 60-year oscillation in GMSL, the possibility should be considered. when attempting to interpret the acceleration in the rate of global and regional mean sea level rise (Chambers et al., 2012).

This finding was also supported by parallel climate studies. Scafetta, 2010 for instance determined empirical evidence for a celestial origin of the climate oscillations with 20 and 60 years. More recently Zotov et al, 2016 detected similar quasi 60 and 20-year periodic oscillations in the global mean Earth temperature anomaly (HadCRUT4) and GMSL. Alternatively, Haigh et al., 2011 reported a 59year period generated by the interference of $8.85 \mathrm{yr}$. cycle of lunar perigee with $18.6 \mathrm{yr}$. nodal cycle at global scale.

To evaluate the effect of the 60 -year periodic at the global scale on the estimated global acceleration parameter consider the following offshoot of the kinematic model given by eqn. (7) and eqn. (8)

$$
\begin{aligned}
h_{t}= & h_{t_{0}}+v\left(t-t_{0}\right)+a \frac{1}{2}\left(t-t_{0}\right)^{2}+\alpha \sin \left[\frac{2 \pi}{P}\left(t-t_{0}\right)\right] \\
& +\beta \cos \left[\frac{2 \pi}{P}\left(t-t_{0}\right)\right]+\varepsilon_{t}
\end{aligned}
$$

In this model, $P$ denotes the 60 -year global oscillation detected by Chambers et al., (2012). The coefficients $\alpha$ and $\beta$ are to be estimated through which the amplitude and the phase of the periodicity are calculated together with their standard errors. All the other model parameters were already defined by eqn. (7) and eqn. (8) together with their descriptions.

We conducted an OLS solution with H-L procedure to estimate the model parameters in the observation equations given by eqn. (11). Not surprisingly, the corresponding normal equations of the observation equations cannot be inverted because of the collinearity of the acceleration parameter and both components of the 60 -year oscillation. Hence, 60-year periodic sea level oscillation cannot be separated from the global sea level acceleration. Nevertheless, an alternative model solution without the acceleration parameter is possible. We carried out another OLS solution using H-L procedure without the acceleration parameter. The estimated model parameters of this model using H-L procedure are listed in Table 1 as Solution $\mathrm{V}$, which only admits intercept, trend and cos component of the 60-year oscillation parameters as statistically significant. The standard errors (a posteriori variance of unit weights) of this solution without the acceleration parameters compared to the model solutions with the accel- eration parameter are the same, 1.06. Moreover, their adjusted $\mathrm{R}^{2}$ values of $71.49 \%$ and $71.52 \%$ for the Solution $\mathrm{V}$ and Solution III, respectively, are indistinguishable from each other. Consequently, the solution that is obtained using the model with the 60 -yr period but without the acceleration parameter is as good as the solution with the acceleration parameter only.

Unfortunately, there are more confounders that will also obscure the declared global acceleration by Nerem at al., (2018) and Ablain et al., (2019).

\section{More confounders: Compounding of luni-solar harmonics}

The 60-year cycle in GMSL variations is not unique. Jevrejeva, et al., (2006) for instance reported nonlinear trends and multiyear cycles in sea level records up to 30 years by examining TG time series. The origins of these global oscillations are multi-causal, and their signatures can also be multi decadal. Among them, some of the effects are of astronomical, such as of lunar solar origin. The interactions of the deterministic components of sea level variations with those from natural ocean and atmosphere interactions are potentially demonstrable via a simple trigonometric identity, which shows that the sum of two sinusoids with angular frequencies $\omega_{A}$ and $\omega_{B}$ is equivalent to the multiplication of sine and cosine functions with two different angular frequencies,

$$
\sin \left(\omega_{A} t\right)+\sin \left(\omega_{B} t\right)=2 \sin \left(\frac{\omega_{A}+\omega_{B}}{2} t\right) \cos \left(\frac{\omega_{A}-\omega_{B}}{2} t\right)
$$

where the angular frequencies are related to their corresponding periods $P_{A}$ and $P_{B}$ via,

$$
\omega_{A}:=\frac{2 \pi}{P_{A}}, \quad \omega_{B}:=\frac{2 \pi}{P_{A}}
$$

The resulting sine function's frequency is the average frequency of the composite wave form and the argument of the cosine function is the subharmonic frequency. These are the carrier and modulation angular frequencies respectively, and the latter is at twice the subharmonic angular frequency. It is also called beat frequency. All harmonics, including subharmonics, are the results of sums and differences of the fundamental frequencies.

Such an effect is well-studied by the tidal community and known as compound tides (Schureman, 1940 pgs. 47, 167). Munk et al. (2002) suggested two different exciting mechanisms for compounding in relation to climate 
change; 1) harmonic beating with the necessary conditions for generating subharmonics of the periods $P_{A}$ and $\left.P_{B} ; 2\right)$ repeat coincidences and the necessary conditions due to the events of near-perfect constructive interference that occurs at given intervals (ibid.). Earlier, Keeling and Whorf (1997) also investigated the latter phenomenon. Under these scenarios, interactions of ocean, meteorological forcing, atmospheric, and sea surface temperature materialize as natural broad band sea level variations and modulate orbital forcing, such as lunar node tide. Hence, their interplay leads climate variations at various time scales.

The source of such variations also includes multidecadal scale sea level variations in relation to the regression of the lunar node which completes its cycle in $P=18.613$ yr, to produce subharmonics with periods including: $2 \times P=37.226$ yr.; $3 \times P=55.839$ yr.; $5 \times P=74.452 \mathrm{yr}$., and its super harmonics with periods: $P / 2=9.306 \mathrm{yr}$.; $P / 3=$ 6.204 yr.; ... $P / 8=2.327$ yr.

In line with the hypothesis put forward by Munk et al. (2002), sea level oscillations are originated from coupling of external forcing such as solar radiation (insolation) with other natural or forced sea level variations, due to mostly broadband internal ocean-atmosphere interactions, producing signatures in sea level variations at multi-decadal time scales. Under this scenario, the variations in total solar radiation with a period of $P=11.1 \mathrm{yr}$., will yield subharmonics with periods: $2 \times P=22.2 \mathrm{yr}$. and longer. However, the additional sub or super harmonic beatings due to the solar radiation forcing of the sea level would have periods near the lunar nodal periods, and likely be weaker than the impact of the sea level variation of lunar origin (Currie, 1987).

The compounding of sea level periodic variations and their materializations in sea level variations at multidecadal scales is not only a conjecture. Yndestad et al. (2008), for instance, reported that the water-property timeseries show mean variability correlated to a subharmonic cycle of the nodal tide of about 74 years. They have found significant correlations between dominant Atlantic water temperature cycles and the 18.6-year lunar node tide, and for $P / 2$ = 9.306-year lunar nodal phase tide. An earlier wavelet analysis by Yndestad (2006) identified a number of lunar node sub and super harmonics in Arctic sea level, temperature, ice extent and winter index time series data, including the signature of nodal harmonics in pole position time series (Table 1 in Yndestad, 2008), and a strong cross correlation with Chandler wobble.

Although the observed amplitudes of the 18.6-year nodal constituent are small, around $15-35 \mathrm{~mm}$, compounding of the nodal tide with natural sea level variations can potentially bias sea level trend and acceleration estimates as confounders for short TG and SA time series and thereby hinder the search of a global GMSL acceleration caused by anthropogenic global warming. As early as 2006, İz has quantified theoretically that these periodicities despite their small amplitudes, if not modelled, will bias the sea trend estimates for shorter series. Moreover, their effects may also be mistakenly interpreted as an accelerated sea level rise if they are not incorporated into the models in analyzing short as well as longer series. İz (2014) study revealed statistically significant periodic oscillations at $27 \mathrm{TG}$ stations with long records (Figure 7). Spatially weighted means of the amplitudes of sub and super harmonics and their standards errors at these stations are displayed in Figure 8. These sea level oscillations at globally distributed TG stations like the one with 60 -year periodicity cannot be separated from the GMSL acceleration in globally averaged sea level time series of SA measurement spanning only 26 years as shown in the previous section but can be assessed as an alternative phenomenon in SA based GMSL time series.

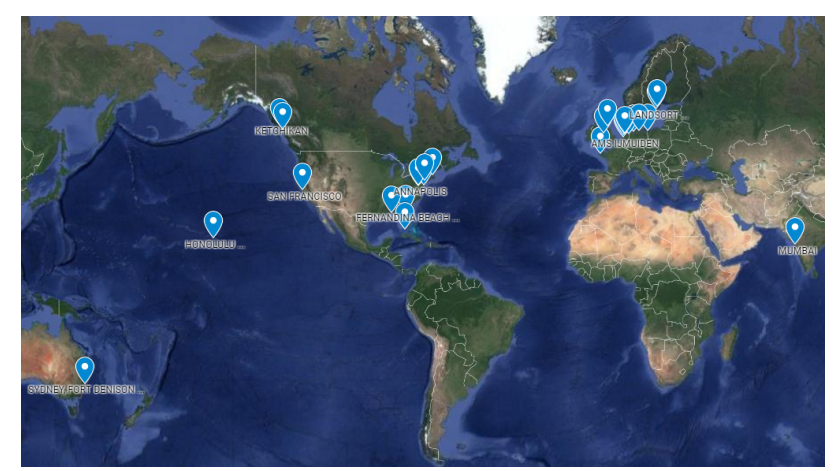

Fig. 7. The locations of the TG stations used in İz (2014) study are indicated with drops (Google maps, 2016).

We carried out three more solutions using OLS (H-L) procedure incorporating into the model for each solution, multidecadal sea level variations with periods 74,56 , and 37-year that were statistically significant at globally distributed TG stations (Figure 8). As expected, multidecadal sea level variations are indistinguishable from a GMSL acceleration because of their collinearity in short-span SA time series. Nonetheless, solutions without an acceleration parameter were possible. The estimated GMSL trends and amplitudes ${ }^{6}$ of the periodicities are listed in Table 1 as Solution VI, VII and VIII using 74, 56, and 37-year periodicities, respectively. The results indicate that the inclusion

6 Only the cosine components of the amplitudes were statistically significant. 


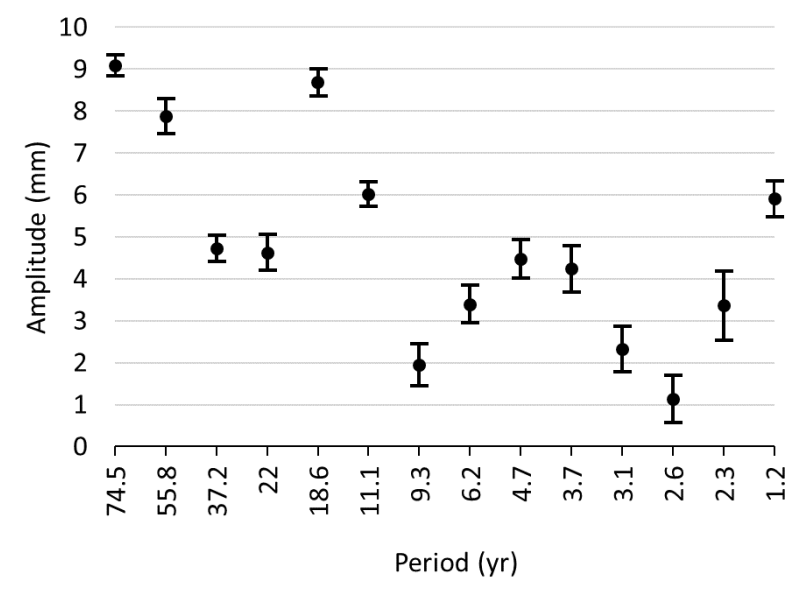

Fig. 8. The amplitudes and the standard errors (error bars) are the weighted averages of the estimated amplitudes across 27 TG stations. All weighted averages are statistically significant. Weights are the inverse variances of the amplitude estimates at each TG station, which are regionally correlated with a spatial correlation of 0.80 among the stations (estimated from their residuals) but not correlated among the regions (íz et al., 2018).

of the multidecadal variations without an acceleration parameter has no effect on the estimated GMSL velocities, their standard errors, and the standard error of the solutions (a posteriori variance of unit weight) compared to the results in the presence of an acceleration only, namely, Solution III. Consequently, any of these models or their combinations thereof, are also competing explanations for the GMSL acceleration.

In addition to the statistically significant multi decadal sea level variations of luni-solar origin as discussed above, another study by İz (2015) revealed that once low frequency GMSL variations are modeled and removed, TG residuals unveiled additional periodic variations at decadal scale, with periods predominantly 12-13 year along the western Atlantic coastal lines. These decadal persistent oscillations are attributed to the ocean surface wind forcing and periodic changes in atmospheric pressure (İz, 2018a) and will serve as additional confounders to be accounted for.

All these confounders, however, do not imply conclusively the absence of an acceleration at global scale for the globally averaged SA time series. For instance, the study by İz et al., (2018) demonstrated the presence of sea level accelerations at globally distributed TG stations with century long data during the SA era (Figure 7). Estimated sea level accelerations at these stations are shown in Figure 8. They were calculated using models that incorporate low frequency, decadal, and multi-decadal periodic sea level variations. The magnitudes of all the estimated accelerations at these TG stations are, nonetheless, substantially lower than the global acceleration estimates reported by Nerem et al. (2018) and Ablain et al. (2019) listed in Table 1.

Hence, one can still choose to opt for a global acceleration model but must account for the omission error for the statistically significant periodic oscillations in GMSL of known origin as detected by TG measurements spanning century long time series. Unfortunately, a statistically significant GMSL acceleration is unlikely to survive such scrutiny. Therefore, for the time being, it would be misleading to declare a global sea level acceleration during the SA era with an acceptable uncertainty and make predictions if the origin of the perceived acceleration is likely to be an unmodeled periodicity or periodicities.

\section{Conclusion}

Recent analyses of SA time series by Nerem at al., (2018) and Ablain et al., (2019) and others declaring a GMSL acceleration are far from exhaustive for such an important topic. Uncertainty is one of the essential components in assessing variability in global sea level in the context of GMSL budget to ascertain anthropogenic contributions. The omission of the effect of potential confounders including a potential jerk or multidecadal sea level variations (Ablain at al., 2019), or using a conjecture that they will average out because of the superior global coverage of SA by Nerem at al. (2018) is a leap of faith without evidence in quantifying a GMSL acceleration and its uncertainty using globally averaged SA time series. Moreover, conducting projections as in Nerem at al. (2018) without ascribing proper uncertainties to the model estimates have no meaning. Until the uncertainty of a recent GMSL acceleration is established in the context of "total evidence", i.e. in the light of systematic global sea level variations during the $20^{\text {th }}$ century revealed by TG measurements together with the available SA time series, any prediction of a GMSL rise ought to be made with extreme prudence.

Acknowledgments: We thank to the three anonymous reviewers for their careful reading the manuscript and their comments. 


\section{References}

Ablain M., Meyssignac B., Zawadzki L., Jugier R., Ribes A., Spada G., Benveniste J., Cazenave A., and Picot N., 2019, Uncertainty in satellite estimate of global mean sea level changes, trend and acceleration, Earth Syst. Sci. Data, 11, 1189-1202.

Bray D., H von Storch, 2009, "Prediction” or "Projection"? The Nomenclature of Climate Science - Science Communication.

Beckley, B., Zelensky, N.P. Holmes, S.A., Lemoine, F.G., Ray, R.D., Mitchum, G.T., Desai, S., Brown, S.T., 2016, Global Mean Sea Level Trend from Integrated Multi-Mission Ocean Altimeters TOPEX/Poseidon Jason-1 and OSTM/Jason-2 Version 4.2. Ver. 4.2. PO.DAAC, CA, USA. Dataset accessed [2018-09-02] at http://dx.doi.org/10.5067/GMSLM-TJ142.

Chambers D.P., M.A. Merrifield, R. S. Nerem, 2012, Is there a 60year oscillation in global mean sea level? Geophysical Research Letters 39:18.

Church, J. A., and N. J. White, 2006, A 20th century acceleration in global sea-level rise, Geophys. Res. Lett., 33, L01602.

Church, J.A., P.U. Clark, A. Cazenave, J.M. Gregory, S. Jevrejeva, A. Levermann, M.A. Merrifield, G.A. Milne, R.S. Nerem, P.D. Nunn, A.J. Payne, W.T. Pfeffer, D. Stammer and A.S. Unnikrishnan, 2013, Sea Level Change. In: Climate Change 2013: The Physical Science Basis. Contribution of Working Group I to the Fifth Assessment Report of the Intergovernmental Panel on Climate Change [Stocker, T.F., D. Qin, G.-K. Plattner, M. Tignor, S.K. Allen, J. Boschung, A. Nauels, Y. Xia, V. Bex and P.M. Midgley (eds.)]. Cambridge University Press, Cambridge, United Kingdom and New York, NY, USA.

Davis, J. L., and N. T. Vinogradova, 2017, Causes of accelerating sea level on the East Coast of North America, Geophys. Res. Lett., 44, 5133-5141.

Dieng, H. B., A. Cazenave, B. Meyssignac, and M. Ablain, 2017, New estimate of the current rate of sea level rise from a sea level budget approach, Geophys. Res. Lett., 44, 3744-3751.

GSFC., 2017, Global Mean Sea Level Trend from Integrated Multi-Mission Ocean Altimeters TOPEX/Poseidon, Jason-1, OSTM/Jason-2 Version 4.2 Ver. 4.2 PO.DAAC, CA, USA. Dataset accessed [2018-09-01] at http://dx.doi.org/10.5067/GMSLMTJ42.

Haigh I.D., Eliot M, Pattiaratchi C., 2011, Global influences of the 18.61 year nodal cycle and 8.85 year cycle of lunar perigee on high tidal levels. J. Geophys. Res. Oceans. 2011;116:C06025.

Hildreth, G. and Lu T., 1960, Demand relations with autocorrelated disturbances, Technical Bulletin 276, Michigan State University Agricultural Experiment.

Houston J.R. and R.G. Dean, 2011, Sea-Level Acceleration Based on U.S. Tide Gauges and Extensions of Previous Global-Gauge Analyses, Journal of Coastal Research, 27, 409 - 417.

IPCC 2007 Summary for Policymakers. In: Climate Change, 2007, The Physical Science Basis. Contribution of Working Group I to the Fourth Assessment Report of the Intergovernmental Panel on Climate Change, [Solomon, S., D. Qin, M. Manning, Z. Chen, M. Marquis, K.B. Averyt, M.Tignor and H.L. Miller (eds.)]. Cambridge University Press, Cambridge, United Kingdom and New York, NY, USA.

İz H.B., T.Y. Yang, C.K. Shum, C.Y. Kuo, 2019, An optimal statistical model to estimate vertical crustal movements us- ing satellite altimetry and tide gauge data (preprint: DOI: 10.13140/RG.2.2.16786.40644).

İz H.B., C.K. Shum, C.Y. Kuo, 2018, Sea Level Accelerations at Globally Distributed Tide Gauge Stations During the Satellite Altimetry Era, J. Geod. Sci. Vol. 8, pp. 130-135.

İz H.B., 2018, Why and How to Predict Sea Level Changes at a Tide Gauge Station with Prediction Intervals, J. Geod. Sci. Vol. 8, pp. 121-129.

İz H.B., 2018a, The effect of regional sea level atmospheric pressure to sea level variations at globally distributed tide gauge stations with long records, J. Geod. Sci. Vol. 8, pp. 55-71.

İ H.B., 2017, Acceleration of the Global Coastal Sea Level Rise During the 20th Century Re-evaluated, J. Geod. Sci., Vol. 7, pp. 51-58.

İz H.B., 2016a, Thermosteric contribution of warming oceans to the global sea level variations, J. Geod. Sci., Vol. 6, pp. 130-138.

İz H.B., 2016b, The effect of warming oceans at a tide gauge station, J. of Geod. Sci., Vol. 6, pp. 69-79.

İz H.B., 2015, More confounders at global and decadal scales in detecting recent sea level accelerations, J. of Geod. Sci, 5, 192198.

İ H.B., 2014, Sub and super harmonics of the lunar nodal tides and the solar radiative forcing in global sea level changes, J. of Geod. Sci., 4, 150-165.

İ H.B., X.L. Ding, and C.K. Shum, 2013, Global Sea Level Trends in the Presence of Variable Sea Level Velocities, and Variable Accelerations, J. Geod. Sci., Vol. 3, Issue 2, pp. 127-135

İz H.B., L. Berry, and M. Koch, 2012, Modeling regional sea level rise using local tide gauge data, J. of Geod. Sci., Vol. 2, Issue 3, pp. 188-1999.

İz H.B., 2006, How do Unmodeled Systematic MSL Variations Affect Long Term Sea Level Trend Estimates from Tide Gauge Data? Journal of Geodesy, Vol. 80, No .1, pp. 40-46.

Jevrejeva, S., A. Grinsted, J. C. Moore, and S. Holgate, 2006, Nonlinear trends and multiyear cycles in sea level records, J. Geophys. Res., 111, C09012, doi:10.1029/2005JC003229.

Keeling, C. D., and T. P. Whorf, 1997, Possible forcing of global temperature by oceanic tides. Proc. Natl. Acad. Sci., 94, 83218328.

Munk, W., M. Dzieciuch, S. Jayne, 2002, Millennial Climate Variability: Is There a Tidal Connection? J. Climate, 15, 370-385.

Nerem R. S., B. D. Beckley, J. T. Fasullo, B. D. Hamlington, D. Masters and G. T. Mitchum, 2018, Climate-change-driven accelerated sea-level rise detected in the altimeter era. PNAS, 1-4.

Neter J.M., Kutner H., Nachtsheim C. J, and Wasserman M., 1996, Applied linear statistical models, Richard D. Irwin, 1408.

NOAA ESRL Physical Sciences Division, 2019, https://www.esrl.noaa.gov/psd/about/, Accessed in October 2019.

Scafetta N., 2010, Empirical evidence for a celestial origin of the climate oscillations and its implications, Journal of Atmospheric and Solar-Terrestrial Physics, Vol.72, pp. 951-970.

Schureman P., 1940, Manual of Harmonic Analysis and Prediction of Tides U. S. Department of Commerce, Washington, pgs. 317.

Yi, S., W. Sun, K. Heki, and A. Qian, 2015, An increase in the rate of global mean sea level rise since 2010 , Geophys. Res. Lett., 42, 1-9.

Yndestad H., 2006, The influence of the lunar nodal cycle on Arctic climate. ICES. Journal of Marine Science, 63: 401- 420. 
Yndestad H., W.R. Turrell, V. Ozhigin, 2008, Lunar nodal tide effects on variability of sea level, temperature, and salinity in the Faroe-Shetland Channel and the Barents Sea, Deep Sea Research Part I: Oceanographic Research Papers, Vol. 55, Issue 10, October 2008, pp 1201-1217.

Zotov L., C.Bizouard, C.K.Shum, 2016, A possible interrelation between Earth rotation and climatic variability at decadal timescale Geodesy and Geodynamics, Volume 7, Issue 3. 